\title{
Use of dietary supplements in pregnant women in relation to sociodemographic factors - a report from The Environmental Determinants of Diabetes in the Young (TEDDY) study
}

\author{
Carin Andrén Aronsson ${ }^{1, *}$, Kendra Vehik ${ }^{2}$, Jimin Yang $^{2}$, Ulla Uusitalo ${ }^{2}$, Kristen Hay ${ }^{3}$, \\ Gesa Joslowski $^{4}$, Anne Riikonen ${ }^{5}$, Lori Ballard ${ }^{2}$, Suvi M Virtanen ${ }^{5,6}$ and Jill M Norris", \\ on behalf of the TEDDY Study Groupt \\ 'Department of Clinical Sciences, Lund University, CRC, Bldg. 60:1 1, Jan Waldenströms gata 35, SE 20502 \\ Malmö, Sweden: ${ }^{2}$ Pediatrics Epidemiology Center, University of South Florida, Tampa, FL, USA: ${ }^{3}$ Pacific \\ Northwest Diabetes Research Institute, Seattle, WA, USA: ${ }^{4}$ Research Institute of Child Nutrition, Dortmund, \\ Germany: ${ }^{5}$ National Institute for Health and Welfare, Nutrition Unit, Helsinki, Finland: ${ }^{6}$ Science Center of \\ Pirkanmaa Hospital District, Tampere, Finland: ${ }^{7}$ Colorado School of Public Health, University of Colorado, \\ Denver, CO, USA
}

Submitted 9 July 2012: Final revision received 22 November 2012: Accepted 17 January 2013: First published online 4 March 2013

\begin{abstract}
Objectives: The aim of the present study was to examine the prevalence and associated factors of dietary supplement use, particularly supplements containing vitamin D and fatty acids, in pregnant women enrolled in a multi-national study. Design: The Environmental Determinants of Diabetes in the Young (TEDDY) study is a prospective longitudinal cohort study. Maternal dietary supplement use was self-reported through questionnaires at month 3 to 4 postpartum.

Setting: Six clinical research centres; three in the USA (Colorado, Georgia/Florida and Washington) and three in Europe (Sweden, Finland and Germany).

Subjects: Mothers ( $n$ 7326) to infants screened for high-risk HLA-DQ genotypes of type 1 diabetes.

Results: Ninety-two per cent of the 7326 women used one or more types of supplement during pregnancy. Vitamin D supplements were taken by $65 \%$ of the women, with the highest proportion of users in the USA (80.5\%). Overall, $16 \%$ of the women reported taking fatty acid supplements and a growing trend was seen in all countries between 2004 and $2010(P<0 \cdot 0001)$. The use was more common in Germany (32\%) and the USA (24\%) compared with Finland (8.5\%) and Sweden $(7 \cdot 0 \%)$. Being pregnant with the first child was a strong predictor for any supplement use in all countries. Low maternal age $(<25$ years), higher education, $\mathrm{BMI} \geq 25 \cdot 0 \mathrm{~kg} / \mathrm{m}^{2}$ and smoking during pregnancy were factors associated with supplement use in some but not all countries.

Conclusions: The majority of the women used dietary supplements during pregnancy. The use was associated with sociodemographic and behavioural factors, such as parity, maternal age, education, BMI and maternal smoking.
\end{abstract}

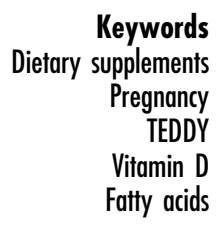

Dietary supplements contribute to the total nutrient intake in pregnant women. It has been reported that among women using supplements containing vitamin D, more than $50 \%$ of the total vitamin D intake is provided by the supplement ${ }^{(1,2)}$ and that vitamin $\mathrm{D}$ and fatty acids from food and supplements are significantly correlated with maternal and cord blood serum levels ${ }^{(2,3)}$. Further, high intake of vitamin $\mathrm{D}$ and $n-3$ fatty acids from both foods and dietary supplements during pregnancy has been reported to be associated with reduced risk of islet autoimmunity and development of type 1 diabetes

$\dagger$ Members of the TEDDY Study Group are listed in Appendix 1.
(T1D) in the offspring ${ }^{(4,5)}$. It is hypothesized that the immune-regulatory properties of vitamin D and the antiinflammatory properties of the long-chain $n$ - 3 fatty acids may inhibit the pathogenesis of $\mathrm{T}^{\mathrm{D}} \mathrm{D}^{(6,7)}$.

Pregnant women are encouraged to enhance their diet during pregnancy to meet increased needs for most nutrients. There are no explicit recommendations regarding consumption of dietary supplements, but women with poor diets, iron-deficiency anaemia, vegans, smokers and women carrying two or more fetuses are recommended to enhance their diet with a prenatal dietary supplement ${ }^{(8)}$. The prevalence of supplement use during pregnancy has been reported to be as high as $80 \%{ }^{(1,2,9)}$. However, there 
are few such studies, especially in the USA ${ }^{(10)}$. The National Health and Nutrition Examination Survey (NHANES) has monitored the use of dietary supplements in the US population since the 1970s, but the sample size of pregnant women is too small to draw conclusions regarding dietary supplement use during pregnancy among US women ${ }^{(11)}$. Supplement use is also reported to be related to sociodemographic variables ${ }^{(10,12)}$. NHANES showed that dietary supplement use in the adult population varied by education level, weight status and ethnicity ${ }^{(11)}$. Supplement use during pregnancy appears to vary among ethnic and socio-economic groups and interact with factors such as supplement use before pregnancy and smoking ${ }^{(10)}$. Supplement use during pregnancy has been reported to be related to age, education, smoking and BMI in the Nordic countries $^{(1,9,13,14)}$.

The primary aim of the present study was to describe the prevalence of dietary supplement use, and particularly the use of supplements containing vitamin D and fatty acids, in pregnant women whose children were at increased genetic risk of T1D. We also wanted to identify sociodemographic and behavioural factors associated with supplement use.

\section{Materials and methods}

\section{Participants}

The Environmental Determinants of Diabetes in the Young (TEDDY) is a prospective study designed to examine dietary and environmental exposures on islet autoimmunity and the development of T1D among children with high-risk HLA-DQ genotypes in the USA, Finland, Germany and Sweden ${ }^{(15)}$. Infants are followed from birth until T1D is diagnosed or until the child is 15 years old. A total of 424788 newborns were screened between September 2004 and February 2010, and 8677 HLA eligible infants were enrolled in the study ${ }^{(16)}$. Ongoing monitoring for diet, infectious agents, other environmental exposures and autoimmunity status are completed with interviews, questionnaires, food records and laboratory tests. Detailed study design and methods have been previously published ${ }^{(17)}$.

Information on dietary supplement consumption was asked at month 3 to 4 postpartum from 8677 women. The following women were excluded: (i) mothers of 850 children (9.8\%) who had not completed the 9-month clinic visit, where all sociodemographic data were collected; (ii) mothers of sixty-six children $(0 \cdot 8 \%)$ with missing supplement use data; (iii) mothers of 178 children $(2 \cdot 1 \%)$ due to missing or extreme anthropometric measures (BMI $<$ 16.0 or $>50.0 \mathrm{~kg} / \mathrm{m}^{2}$ ); and (iv) mothers of 257 children (3.0\%) with incomplete or missing sociodemographic data, resulting in a total of 7326 mothers with complete data. Written informed consents were obtained for all study participants from a parent or primary caregiver, separately, for genetic screening and participation in prospective follow-up. The study was approved by local Institutional Review Boards (six clinical centres in four countries) and is monitored by an External Advisory Board formed by the National Institutes of Health.

\section{Questionnaires}

A questionnaire containing questions on illnesses, medications, smoking habits, alcohol intake, diet and dietary supplement use during pregnancy was mailed to the women of enrolled children and was completed at month 3 to 4 postpartum. Dietary supplements were defined as supplements that contain one or any combination of the following substances: a vitamin, mineral, herb or other botanical substance, and amino acids, in accordance with the US Dietary Supplement Health and Education Act of 1994. Homeopathic and protein supplements were not coded as dietary supplements in TEDDY ${ }^{(18)}$. Dietary supplements taken due to medical condition or illness were also included. The women reported product name, frequency per week and duration of usage during pregnancy. Supplements were classified into twenty-seven single supplements and ten multivitamin/mineral subgroups based on their nutrient profiles (Appendix 2) ${ }^{(19)}$. A dietary supplement user was defined as anyone who reported taking at least one supplement at least once during the pregnancy.

Maternal education was recorded on a ten-category scale to accommodate for different education systems in each country, which was then aggregated into two categories, basic primary education (primary school through trade school) and higher education (completed trade school or higher), to achieve comparability across countries. Maternal age was defined as the woman's age at time of delivery and was treated as both a continuous and a categorical variable $(<25 \cdot 0$ years, 25.0-29.9 years, $30 \cdot 0-34 \cdot 9$ years and $\geq 35 \cdot 0$ years). Pre-pregnancy BMI was calculated by using mother's self-reported weight (in kilograms) before pregnancy divided by the square of height (in metres) and was categorized based on the WHO classification ${ }^{(20)}$. Smoking and alcohol consumption during pregnancy were defined as 'yes' if reported, regardless of frequency and duration. Birth order of the TEDDY child (first-born $v$. others) was determined by the question 'Is this your first child?' Maternal diabetes status during pregnancy was self-reported and was grouped into four groups: 'no diabetes', 'type 1 diabetes', 'gestational diabetes and type 2 diabetes' and 'unknown'. Race/ ethnicity was analysed only in the US women and was categorized in five groups (non-Hispanic white, African Americans, Hispanics, all other races and unknown).

\section{Statistical analyses}

Data were analysed using the SAS ${ }^{\circledR}$ statistical software package version $9 \cdot 2$. Categorical variables were analysed using the Pearson $\chi^{2}$ test or Fisher's exact test and continuous variables were analysed using ANOVA. Multiple logistic regression analysis was used to determine the 
independent factors associated with maternal use of supplements containing vitamin D and fatty acids. All models were assessed overall and separately by country.

\section{Results}

The distribution of characteristics of the study population across the four countries is provided in Table 1. Germany had the highest maternal age (mean 31.6 years) followed by Sweden, the USA and Finland $(P<0 \cdot 0001)$. Pre-pregnancy BMI was higher among US women compared with the European women $(P<0 \cdot 0001)$. Finland had the highest proportion of women with higher education followed by Germany, the USA and Sweden $(P<0 \cdot 0001)$. A greater proportion of German women reported smoking and drinking during pregnancy than women from the other countries $(P<0 \cdot 0001)$. The use of dietary supplements during pregnancy was common among women in all countries (Table 2). The highest percentage of users was in the USA (96.4\%) while the lowest was in Finland (87.1\%) Users tended to be older women ( $\geq 35 \cdot 0$ years), to be pregnant with their first child and to have a higher educational level compared with non-users. In both the USA and Finland, drinking alcohol during pregnancy was more common among supplement users than non-users, although these differences were not seen in Germany and Sweden. Smoking was more often reported among the non-users in all four countries. A higher percentage of women with $\mathrm{BMI} \geq 25 \cdot 0$ $\mathrm{kg} / \mathrm{m}^{2}$ was seen among non-users in Germany $(P=0 \cdot 0003)$ and Sweden $(P<0 \cdot 0001)$ but not in the USA and Finland.

\section{Predictors of any dietary supplement use during pregnancy}

Results of the multivariable analysis demonstrated that in all countries, women who were pregnant with their first child were more likely to use supplements (Table 3). Younger women $(<25 \cdot 0$ years $)$ were less likely to use supplements compared with older women. Women with higher education in the USA and Sweden, but not in Finland and Germany, were more likely to use dietary supplements.

Table 1 Cohort characteristics by country ( $n$ 7326): The Environmental Determinants of Diabetes in the Young (TEDDY) study

\begin{tabular}{|c|c|c|c|c|c|c|c|c|c|}
\hline & \multicolumn{2}{|c|}{$\begin{array}{c}\text { USA } \\
(n \text { 2961) }\end{array}$} & \multicolumn{2}{|c|}{$\begin{array}{l}\text { Sweden } \\
(n \text { 2231) }\end{array}$} & \multicolumn{2}{|c|}{$\begin{array}{l}\text { Finland } \\
\text { (n 1622) }\end{array}$} & \multicolumn{2}{|c|}{$\begin{array}{l}\text { Germany } \\
(n 512)\end{array}$} & \\
\hline & Mean & SD & Mean & SD & Mean & SD & Mean & SD & \\
\hline \multirow{3}{*}{$\begin{array}{l}\text { Maternal age (years) } \\
\text { Pre-pregnancy BMI }\left(\mathrm{kg} / \mathrm{m}^{2}\right)\end{array}$} & $30 \cdot 6^{b}$ & $5 \cdot 7$ & $30 \cdot 8^{b}$ & $4 \cdot 6$ & $30 \cdot 5^{c}$ & $5 \cdot 0$ & $31 \cdot 6^{\mathrm{a}}$ & $4 \cdot 9$ & \\
\hline & $25 \cdot 6^{\mathrm{a}}$ & $6 \cdot 0$ & $24 \cdot 3^{b}$ & $4 \cdot 6$ & $24 \cdot 2^{b}$ & $4 \cdot 6$ & $24 \cdot 2^{b}$ & $4 \cdot 9$ & \\
\hline & $n$ & $\%$ & $n$ & $\%$ & $n$ & $\%$ & $n$ & $\%$ & $P$ \\
\hline Maternal education & & & & & & & & & $<0.0001$ \\
\hline Basic primary & 483 & $16 \cdot 3$ & 751 & $33 \cdot 7$ & 157 & $9 \cdot 7$ & 70 & $13 \cdot 7$ & \\
\hline Higher education & 2478 & $83 \cdot 7$ & 1480 & $66 \cdot 3$ & 1465 & $90 \cdot 3$ & 442 & $86 \cdot 3$ & \\
\hline Mother's first child & & & & & & & & & $<0.0001$ \\
\hline Yes & 1247 & $42 \cdot 1$ & 1064 & $47 \cdot 7$ & 731 & $45 \cdot 1$ & 259 & $50 \cdot 6$ & \\
\hline No & 1714 & $57 \cdot 9$ & 1167 & $52 \cdot 3$ & 891 & $54 \cdot 9$ & 253 & $49 \cdot 4$ & \\
\hline Pre-pregnancy BMI (kg/m²) & & & & & & & & & $<0.0001$ \\
\hline$<18.5$ & 86 & $2 \cdot 9$ & 64 & $2 \cdot 9$ & 54 & $3 \cdot 3$ & 26 & $5 \cdot 1$ & \\
\hline $18 \cdot 5-24 \cdot 9$ & 1604 & $54 \cdot 2$ & 1398 & $62 \cdot 7$ & 1042 & $64 \cdot 2$ & 316 & $61 \cdot 7$ & \\
\hline$\geq 25 \cdot 0$ & 1271 & $42 \cdot 9$ & 769 & $34 \cdot 4$ & 526 & $32 \cdot 4$ & 170 & $33 \cdot 2$ & \\
\hline Smoking during pregnancy & & & & & & & & & $<0.0001$ \\
\hline Yes & 294 & $9 \cdot 9$ & 276 & $12 \cdot 4$ & 227 & $14 \cdot 0$ & 104 & $20 \cdot 3$ & \\
\hline No & 2667 & $90 \cdot 1$ & 1955 & $87 \cdot 6$ & 1395 & $86 \cdot 0$ & 408 & $79 \cdot 7$ & \\
\hline Drinking during pregnancy & & & & & & & & & $<0.0001$ \\
\hline Yes & 1164 & $39 \cdot 3$ & 624 & $28 \cdot 0$ & 493 & $30 \cdot 4$ & 237 & $46 \cdot 3$ & \\
\hline No & 1797 & $60 \cdot 7$ & 1607 & $72 \cdot 0$ & 1129 & $69 \cdot 6$ & 275 & $53 \cdot 7$ & \\
\hline Maternal diabetes status during pregnancy & & & & & & & & & $<0.0001$ \\
\hline None & 2644 & $89 \cdot 3$ & 2092 & $93 \cdot 8$ & 1336 & $93 \cdot 8$ & 344 & $67 \cdot 2$ & \\
\hline T1D & 102 & $3 \cdot 4$ & 39 & $1 \cdot 8$ & 61 & $3 \cdot 8$ & 94 & $18 \cdot 4$ & \\
\hline Gestational or T2D & 167 & $5 \cdot 7$ & 68 & $3 \cdot 0$ & 175 & $10 \cdot 8$ & 21 & $4 \cdot 1$ & \\
\hline Unknown/missing & 48 & $1 \cdot 6$ & 32 & $1 \cdot 4$ & 50 & $3 \cdot 0$ & 53 & $10 \cdot 3$ & \\
\hline TEDDY child with T1D in FDR & & & & & & & & & $<0.0001$ \\
\hline Yes & 327 & $11 \cdot 0$ & 144 & $6 \cdot 5$ & 147 & $9 \cdot 1$ & 196 & $38 \cdot 3$ & \\
\hline No & 2634 & $89 \cdot 0$ & 2087 & $93 \cdot 5$ & 1475 & $90 \cdot 9$ & 316 & $61 \cdot 7$ & \\
\hline Race/ethnicity* & & & & & & & & & \\
\hline Non-Hispanic white & 2193 & $74 \cdot 0$ & & & & & & & \\
\hline African Americans & 62 & $2 \cdot 1$ & & & & & & & \\
\hline Hispanic & 508 & $17 \cdot 2$ & & & & & & & \\
\hline All other & 130 & $4 \cdot 4$ & & & & & & & \\
\hline Unknown/missing & 68 & $2 \cdot 3$ & & & & & & & \\
\hline
\end{tabular}

T1D, type 1 diabetes; T2D, type 2 diabetes; FDR, first-degree relative (mother, father and/or siblings).

${ }_{a, b, c}$ Mean values within a row with unlike superscript letters were significantly different $(P<0.05)$.

*US mothers only. 
Table 2 Distribution and description of non-users and any supplement users, by respondent characteristics: The Environmental Determinants of Diabetes in the Young (TEDDY) study

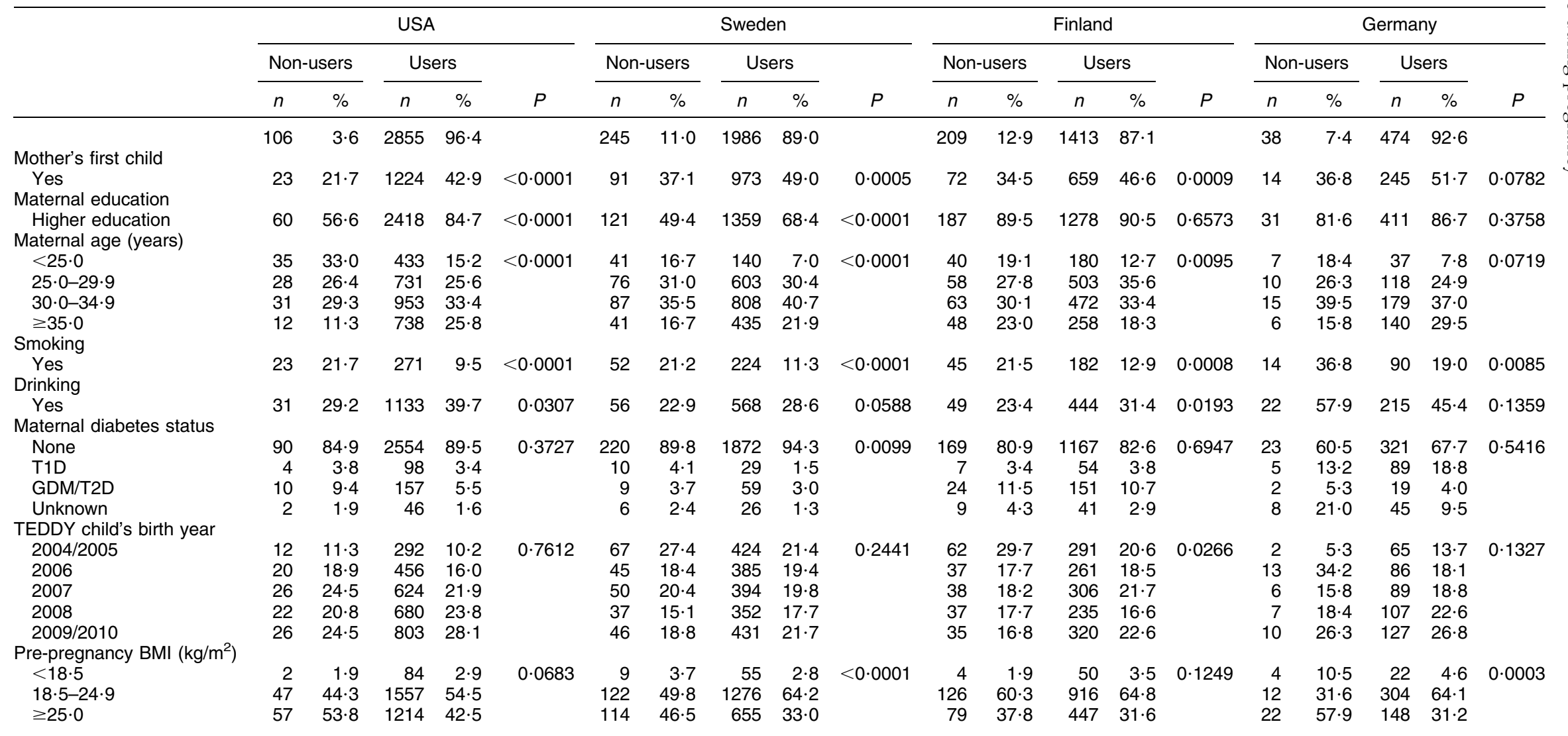

T1D, type 1 diabetes; T2D, type 2 diabetes. 
Table 3 Adjusted sociodemographic predictors for any dietary supplement use during pregnancy, by country: The Environmental Determinants of Diabetes in the Young (TEDDY) study

\begin{tabular}{|c|c|c|c|c|c|c|c|c|c|c|c|c|}
\hline & \multicolumn{3}{|c|}{ USA } & \multicolumn{3}{|c|}{ Sweden } & \multicolumn{3}{|c|}{ Finland } & \multicolumn{3}{|c|}{ Germany } \\
\hline & OR & $95 \% \mathrm{Cl}$ & $P$ & OR & $95 \% \mathrm{Cl}$ & $P$ & OR & $95 \% \mathrm{Cl}$ & $P$ & OR & $95 \% \mathrm{Cl}$ & $P$ \\
\hline \multicolumn{13}{|l|}{ Mother's first child } \\
\hline No & 1.00 & & & 1.00 & & & 1.00 & & & $1 \cdot 00$ & & \\
\hline Yes & $3 \cdot 18$ & $1 \cdot 94,5 \cdot 21$ & $<0.0001$ & 1.98 & $1 \cdot 46,2 \cdot 70$ & $<0.0001$ & $1 \cdot 75$ & $1 \cdot 26,1 \cdot 45$ & 0.0008 & $3 \cdot 18$ & $1 \cdot 40,7 \cdot 25$ & 0.0058 \\
\hline \multicolumn{13}{|l|}{ Maternal education } \\
\hline Basic primary & 1.00 & & & 1.00 & & & 1.00 & & & 1.00 & & \\
\hline Higher education & $2 \cdot 39$ & $1 \cdot 47,3 \cdot 89$ & 0.0005 & $1 \cdot 62$ & $1 \cdot 20,2 \cdot 17$ & 0.0015 & 0.90 & $0.54,1.48$ & NS & $1 \cdot 24$ & $0.45,3 \cdot 41$ & NS \\
\hline \multicolumn{13}{|l|}{ Maternal age (years) } \\
\hline$<25 \cdot 0$ & 0.56 & $0.32,0.97$ & 0.0385 & 0.47 & $0.29,0.74$ & 0.0013 & 0.52 & $0.33,0.83$ & 0.0193 & 0.40 & $0 \cdot 12,1 \cdot 37$ & NS \\
\hline $25 \cdot 0-29 \cdot 9$ & 1.00 & & & $1 \cdot 00$ & & & $1 \cdot 00$ & & & $1 \cdot 00$ & & \\
\hline $30 \cdot 0-34 \cdot 9$ & 1.03 & $0.60,1 \cdot 76$ & NS & $1 \cdot 16$ & $0.82,1.63$ & NS & 0.90 & $0 \cdot 61,1 \cdot 32$ & NS & $1 \cdot 27$ & $0 \cdot 51,3 \cdot 18$ & NS \\
\hline$\geq 35.0$ & $2 \cdot 14$ & $1 \cdot 06,4 \cdot 34$ & 0.0347 & $1 \cdot 43$ & $0.94,2 \cdot 18$ & NS & 0.71 & $0 \cdot 46,1 \cdot 10$ & NS & $2 \cdot 31$ & $0 \cdot 75,7 \cdot 10$ & NS \\
\hline \multicolumn{13}{|c|}{ Pre-pregnancy BMI $\left(\mathrm{kg} / \mathrm{m}^{2}\right)$} \\
\hline$<18.5$ & $1 \cdot 84$ & $0 \cdot 43,7 \cdot 86$ & NS & $0 \cdot 71$ & $0.33,1.52$ & NS & $2 \cdot 08$ & $0 \cdot 73,5 \cdot 96$ & NS & $0 \cdot 24$ & $0.07,0.91$ & 0.0352 \\
\hline $\begin{array}{l}18 \cdot 5-24 \cdot 9 \\
\geq 25 \cdot 0\end{array}$ & $\begin{array}{l}1.00 \\
0.76\end{array}$ & $0.50,1 \cdot 14$ & NS & $\begin{array}{l}1.00 \\
0.64\end{array}$ & $0.48,0.84$ & 0.0017 & $\begin{array}{l}1.00 \\
0.82\end{array}$ & $0 \cdot 60,1 \cdot 12$ & NS & $\begin{array}{l}1.00 \\
0.22\end{array}$ & $0 \cdot 10,0.48$ & 0.0001 \\
\hline \multicolumn{13}{|c|}{ TEDDY child with T1D in FDR } \\
\hline No & 1.00 & & & $1 \cdot 00$ & & & 1.00 & & & $1 \cdot 00$ & & \\
\hline Yes & 1.56 & $0 \cdot 71,3 \cdot 43$ & NS & 0.60 & $0.37,0.96$ & 0.0331 & $3 \cdot 44$ & $1 \cdot 11,10 \cdot 70$ & 0.0328 & $2 \cdot 00$ & $0.87,4 \cdot 60$ & NS \\
\hline \multicolumn{13}{|c|}{ Smoking during pregnancy } \\
\hline No & 1.00 & & & $1 \cdot 00$ & & & 1.00 & & & 1.00 & & \\
\hline Yes & 0.65 & $0 \cdot 38,1 \cdot 10$ & NS & 0.67 & $0.46,0.97$ & 0.0326 & 0.56 & $0.38,0.83$ & 0.0036 & 0.65 & $0 \cdot 29,1 \cdot 47$ & NS \\
\hline \multicolumn{13}{|c|}{ Drinking during pregnancy } \\
\hline No & 1.00 & & & $1 \cdot 00$ & & & 1.00 & & & 1.00 & & \\
\hline Yes & $1 \cdot 14$ & $0 \cdot 73,1 \cdot 78$ & NS & $1 \cdot 18$ & $0 \cdot 85,1 \cdot 64$ & NS & 1.54 & $1 \cdot 08,2 \cdot 19$ & 0.0160 & 0.48 & $0.27,1.02$ & NS \\
\hline \multicolumn{13}{|c|}{ TEDDY child's birth year } \\
\hline $2004 / 2005$ & 1.00 & & & 1.00 & & & 1.00 & & & 1.00 & & \\
\hline 2006 & 0.99 & $0 \cdot 47,2 \cdot 11$ & NS & $1 \cdot 35$ & $0.89,2.04$ & NS & $1 \cdot 42$ & $0.90,2 \cdot 23$ & NS & 0.19 & $0.04,0.92$ & 0.0394 \\
\hline 2007 & 1.05 & $0.51,2 \cdot 17$ & NS & $1 \cdot 29$ & $0.86,1.93$ & NS & 1.84 & $1 \cdot 18,2 \cdot 88$ & 0.0069 & 0.42 & $0.08,2 \cdot 31$ & NS \\
\hline 2008 & $1 \cdot 41$ & $0.67,2.96$ & NS & 1.58 & $1 \cdot 02,2 \cdot 44$ & 0.0420 & $1 \cdot 49$ & $0 \cdot 94,2 \cdot 36$ & NS & 0.58 & $0 \cdot 11,3.07$ & NS \\
\hline $2009 / 2010$ & $1 \cdot 45$ & $0 \cdot 70,2.97$ & NS & 1.53 & $1 \cdot 01,2 \cdot 30$ & 0.0431 & $2 \cdot 27$ & $1 \cdot 42,3 \cdot 63$ & 0.0006 & 0.46 & $0 \cdot 09,2 \cdot 33$ & NS \\
\hline \multicolumn{13}{|l|}{ Race/ethnicity* } \\
\hline Non-Hispanic white & 1.00 & & & & & & & & & & & \\
\hline African Americans & 0.78 & $0.26,2 \cdot 32$ & NS & & & & & & & & & \\
\hline Hispanic & 0.80 & $0.49,1 \cdot 30$ & NS & & & & & & & & & \\
\hline All other & 0.60 & $0.24,1.44$ & NS & & & & & & & & & \\
\hline Missing/unknown & 0.71 & $0 \cdot 21,2 \cdot 43$ & NS & & & & & & & & & \\
\hline
\end{tabular}


Women who smoked during pregnancy were less likely to use dietary supplements in Finland and Sweden, but not in the USA and Germany. In Finland, women were more likely to be supplement users if the child had a first-degree relative diagnosed with T1D or if they reported alcohol consumption during pregnancy. Women in Sweden and Germany with a pre-pregnancy BMI $\geq$ $25 \cdot 0 \mathrm{~kg} / \mathrm{m}^{2}$ were less likely to use any dietary supplement during pregnancy. Race/ethnicity among US women did not predict dietary supplement use.

\section{Use of supplements containing vitamin $D$ or fatty acids}

The overall proportion of women using supplemental vitamin $\mathrm{D}$ from either single or multivitamin preparations was $65 \%$ (4768/7326) and of those, 62\% (2972/4768) used vitamin D supplements throughout the entire pregnancy. The remaining women (38\%) were using supplements on average for $24 \cdot 7$ (sD 10.2) weeks during pregnancy. In the USA, $81 \%$ of the women reported using vitamin D-containing supplements while the proportion of women using supplements was lower in the European countries, with $71 \%$ in Finland, $48 \%$ in Sweden and 33\% in Germany (Table 4). For dietary supplements containing fatty acids, the overall proportion of users was 16\% (1161/7326) and of those, 53\% (616/1161) used the fatty acid supplements throughout the entire pregnancy. The mean duration for the remaining $47 \%$ was 21.3 (SD 9.9) weeks. The highest proportion of fatty acid supplement users was found in Germany (32\%). In the USA, $24 \%$ of the women used such supplements compared with less than 10\% in Finland and Sweden (Table 5).

The prevalence of vitamin D supplement use during pregnancy was relatively stable through the study screening years (2004-2010), except that it increased from 63\% to $79 \%$ in Finland $(P<0.0001$; Fig. 1$)$. Meanwhile, consumption of fatty acid supplements grew in all countries during this 5 -year period $(P<0 \cdot 0001)$. The greatest change was seen in the USA and Germany, with an increase in supplement use by $60 \%$.

\section{Predictors of supplements containing vitamin D during pregnancy}

Being pregnant with the first child was a common predictor for use of vitamin D-containing supplements in all TEDDY countries (Table 4). Higher education in the USA and Sweden, but not in Finland and Germany, was a predictor for vitamin D use. Higher maternal age $(\geq 30 \cdot 0$ years) was also a predictor for vitamin $\mathrm{D}$ supplement use in the USA and Sweden, but not in Germany; and was a predictor of less use in Finland ( $\geq 35 \cdot 0$ years). Women in Sweden with a pre-pregnancy $\mathrm{BMI} \geq 25 \cdot 0 \mathrm{~kg} / \mathrm{m}^{2}$ were less likely to use vitamin D supplements. Women in the USA, Finland and Germany who smoked during pregnancy were less likely to use vitamin D supplements, while alcohol consumption during pregnancy was not associated with the use of vitamin D supplements. In the
USA, women with race/ethnicity other than non-Hispanic white were less likely to use vitamin D supplements.

\section{Predictors of supplements containing fatty acids during pregnancy}

Older maternal age, $\geq 30 \cdot 0$ years in the USA and Germany and $\geq 35.0$ years in Sweden, was associated with using supplements containing fatty acids (Table 5). Higher maternal education was associated with fatty acid supplements use in the USA only. Women pregnant with the first child or with higher maternal age were more likely to use dietary supplements containing fatty acids, except in Finland. Smoking was a predictor of less use in the USA and Germany but not in Finland and Sweden. Women with BMI $\geq 25 \cdot 0 \mathrm{~kg} / \mathrm{m}^{2}$ in the USA and Finland were less likely to use fatty acid supplements. Birth year of the child was associated with a growing prevalence of fatty acid supplement use in all countries. There was no difference in use by race/ethnicity among the US women.

\section{Discussion}

To the best of our knowledge, the current study is the first international prospective cohort study presenting data on maternal dietary supplement use from four countries. High prevalence of any dietary supplement use, ranging from $87 \%$ in Finland to $96 \%$ in the USA, was observed in pregnant women participating in the TEDDY study. Women who were pregnant with their first child were more likely to take supplements. In both the USA and Sweden, supplement users were more likely to have a higher education. Younger women $(<25 \cdot 0$ years $)$ were less likely to use any supplements during their pregnancy in all countries except in Germany. The association between demographic and lifestyle factors and dietary supplement use during pregnancy observed in the TEDDY population was consistent with the literature ${ }^{(1,9,13,14)}$.

In the present study, supplement use was self-reported by mothers at months 3 to 4 postpartum. The questionnaires were checked by trained study personnel for completeness during the first clinic visit. Self-reported supplement use may be associated with both over- or under-reporting, but studies on dietary supplement use during pregnancy have reported a high correlation between biomarker concentrations and intake estimated by self-reported methods ${ }^{(2,3,21)}$.

A healthy pre-pregnancy BMI has often been associated with maternal supplement use, but we did not see a clear association across countries in our analysis. One reason may be due to different cut-off points. We used the current WHO classification without differentiating between overweight and obesity ${ }^{(20)}$. Other studies have used different cut-off points, often in four levels, that define underweight and normal weight slightly differently and separate overweight $\left(\mathrm{BMI}=25 \cdot 0-30 \cdot 0 \mathrm{~kg} / \mathrm{m}^{2}\right)$ from obese $\left(\mathrm{BMI}>30 \cdot 0 \mathrm{~kg} / \mathrm{m}^{2}\right)^{(1,9,14,22)}$. Our results showed 
Table 4 Adjusted sociodemographic predictors for use of supplements containing vitamin D during pregnancy, by country: The Environmental Determinants of Diabetes in the Young (TEDDY) study

\begin{tabular}{|c|c|c|c|c|c|c|c|c|c|c|c|c|}
\hline \multirow{3}{*}{ Users (single or MVM preparations): } & \multicolumn{3}{|c|}{ USA } & \multicolumn{3}{|c|}{ Sweden } & \multicolumn{3}{|c|}{ Finland } & \multicolumn{3}{|c|}{ Germany } \\
\hline & \multicolumn{3}{|c|}{$n 2383 ; 80 \cdot 5 \%$} & \multicolumn{3}{|c|}{$n$ 1060; $47.5 \%$} & \multicolumn{3}{|c|}{$n 1158 ; 71 \cdot 4 \%$} & \multicolumn{3}{|c|}{$n 167 ; 32 \cdot 6 \%$} \\
\hline & OR & $95 \% \mathrm{Cl}$ & $P$ & OR & $95 \% \mathrm{Cl}$ & $P$ & OR & $95 \% \mathrm{Cl}$ & $P$ & OR & $95 \% \mathrm{Cl}$ & $P$ \\
\hline \multicolumn{13}{|l|}{ Mother's first child } \\
\hline No & 1.00 & & & 1.00 & & & $1 \cdot 00$ & & & 1.00 & & \\
\hline Yes & $1 \cdot 29$ & $1 \cdot 05,1 \cdot 58$ & 0.0160 & $1 \cdot 62$ & $1 \cdot 35,1 \cdot 94$ & $<0.0001$ & $1 \cdot 71$ & $1 \cdot 34,2 \cdot 18$ & $<0.0001$ & $1 \cdot 73$ & $1 \cdot 14,2 \cdot 62$ & 0.0098 \\
\hline \multicolumn{13}{|l|}{ Maternal education } \\
\hline Basic primary & 1.00 & & & $1 \cdot 00$ & & & 1.00 & & & $1 \cdot 00$ & & \\
\hline Higher education & 1.53 & $1 \cdot 18,1 \cdot 98$ & 0.0013 & $1 \cdot 79$ & $1 \cdot 47,2 \cdot 17$ & $<0.0001$ & 1.05 & $0.72,1.54$ & NS & 1.93 & $0.96,3.86$ & NS \\
\hline \multicolumn{13}{|l|}{ Maternal age (years) } \\
\hline$<25 \cdot 0$ & 0.58 & $0.43,0.77$ & 0.0002 & $0 \cdot 70$ & $0 \cdot 48,1 \cdot 01$ & 0.0536 & 0.73 & $0.51,1.05$ & NS & $0 \cdot 72$ & $0.31,1 \cdot 66$ & NS \\
\hline $25 \cdot 0-29 \cdot 9$ & $1 \cdot 00$ & & & $1 \cdot 00$ & & & $1 \cdot 00$ & & & $1 \cdot 00$ & & \\
\hline $30 \cdot 0-34 \cdot 9$ & $1 \cdot 39$ & $1 \cdot 07,1 \cdot 80$ & 0.0149 & $1 \cdot 39$ & $1 \cdot 12,1 \cdot 72$ & 0.0023 & $1 \cdot 10$ & $0.83,1.47$ & NS & 0.92 & $0.56,1.53$ & NS \\
\hline$\geq 35 \cdot 0$ & $1 \cdot 31$ & $0.98,1.75$ & NS & $1 \cdot 30$ & $1 \cdot 08,1.67$ & 0.0435 & 0.67 & $0.49,0.92$ & 0.0143 & 0.94 & $0.55,1.62$ & NS \\
\hline \multicolumn{13}{|l|}{ Pre-pregnancy BMI (kg/m²) } \\
\hline$<18.5$ & $1 \cdot 41$ & $0.78,2 \cdot 55$ & NS & $0 \cdot 82$ & $0.49,1.39$ & NS & $1 \cdot 28$ & $0 \cdot 66,2 \cdot 46$ & NS & $1 \cdot 11$ & $0 \cdot 46,2 \cdot 71$ & NS \\
\hline $18 \cdot 5-24 \cdot 9$ & 1.00 & & & 1.00 & & & 1.00 & & & $1 \cdot 00$ & & \\
\hline$\geq 25 \cdot 0$ & $0 \cdot 88$ & $0.73,1.08$ & NS & $0 \cdot 78$ & $0.65,0.94$ & $0 \cdot 0074$ & 0.83 & $0.66,1.06$ & NS & 0.74 & $0 \cdot 48,1 \cdot 12$ & NS \\
\hline \multicolumn{13}{|l|}{ Smoking during pregnancy } \\
\hline No & $1 \cdot 00$ & & & $1 \cdot 00$ & & & 1.00 & & & $1 \cdot 00$ & & \\
\hline Yes & 0.67 & $0.50,0.90$ & 0.0069 & 0.81 & $0.61,1.07$ & NS & 0.52 & $0.38,0 \cdot 71$ & $<0.0001$ & 0.29 & $0.14,0.58$ & 0.0005 \\
\hline \multicolumn{13}{|l|}{ Drinking during pregnancy } \\
\hline No & $1 \cdot 00$ & & & $1 \cdot 00$ & & & 1.00 & & & $1 \cdot 00$ & & \\
\hline Yes & $1 \cdot 22$ & $0.99,1.49$ & NS & 1.05 & $0.86,1 \cdot 27$ & NS & $1 \cdot 26$ & $0.98,1.62$ & NS & 0.91 & $0.60,1.36$ & NS \\
\hline \multicolumn{13}{|l|}{ TEDDY child's birth year } \\
\hline $2004 / 2005$ & $1 \cdot 00$ & & & $1 \cdot 00$ & & & $1 \cdot 00$ & & & $1 \cdot 00$ & & \\
\hline 2006 & $0 \cdot 78$ & $0.52,1.18$ & NS & 0.78 & $0.60,1.02$ & NS & $1 \cdot 23$ & $0.88,1.73$ & NS & 1.99 & $0.95,4.16$ & NS \\
\hline 2007 & 0.69 & $0 \cdot 47,1.01$ & NS & 0.90 & $0.69,1.17$ & NS & $2 \cdot 28$ & $1 \cdot 62,3.23$ & $<0.0001$ & $2 \cdot 81$ & $1 \cdot 34,5 \cdot 90$ & 0.0156 \\
\hline 2008 & 0.69 & $0 \cdot 47,1 \cdot 01$ & NS & 0.96 & $0.73,1 \cdot 26$ & NS & 1.55 & $1 \cdot 09,2 \cdot 20$ & 0.0152 & $2 \cdot 03$ & $0.98,4 \cdot 20$ & NS \\
\hline $2009 / 2010$ & 0.73 & $0.50,1.06$ & NS & $1 \cdot 22$ & $0.94,1.58$ & NS & $2 \cdot 19$ & $1.56,3.09$ & $<0.0001$ & $1 \cdot 40$ & $0.68,2.90$ & NS \\
\hline \multicolumn{13}{|l|}{ Race/ethnicity* } \\
\hline Non-Hispanic white & 1.00 & & & & & & & & & & & \\
\hline African Americans & 0.50 & $0.28,0.88$ & 0.0163 & & & & & & & & & \\
\hline Hispanic & 0.61 & $0.48,0.77$ & $<0.0001$ & & & & & & & & & \\
\hline All other & $0 \cdot 61$ & $0.40,0.95$ & 0.0272 & & & & & & & & & \\
\hline Missing/unknown & 0.46 & $0.26,0.79$ & 0.0052 & & & & & & & & & \\
\hline
\end{tabular}

MVM, multivitamin/mineral.

Separate multivariate models, including the variables listed above, were run for each country.

Separate multivariate
*US mothers only. 
Table 5 Adjusted sociodemographic predictors for use of supplements containing fatty acids during pregnancy, by country: The Environmental Determinants of Diabetes in the Young (TEDDY) study

\begin{tabular}{|c|c|c|c|c|c|c|c|c|c|c|c|c|}
\hline \multirow{3}{*}{ Users (single or MVM preparations): } & \multicolumn{3}{|c|}{ USA } & \multicolumn{3}{|c|}{ Sweden } & \multicolumn{3}{|c|}{ Finland } & \multicolumn{3}{|c|}{ Germany } \\
\hline & \multicolumn{3}{|c|}{$n 704 ; 23 \cdot 8 \%$} & \multicolumn{3}{|c|}{$n 156 ; 7 \cdot 0 \%$} & \multicolumn{3}{|c|}{$n 138 ; 8.5 \%$} & \multicolumn{3}{|c|}{$n 163 ; 31 \cdot 8 \%$} \\
\hline & OR & $95 \% \mathrm{Cl}$ & $P$ & OR & $95 \% \mathrm{Cl}$ & $P$ & OR & $95 \% \mathrm{Cl}$ & $P$ & OR & $95 \% \mathrm{Cl}$ & $P$ \\
\hline \multicolumn{13}{|l|}{ Mother's first child } \\
\hline No & 1.00 & & & 1.00 & & & 1.00 & & & 1.00 & & \\
\hline Yes & $1 \cdot 79$ & $1 \cdot 49,2 \cdot 15$ & $<0.0001$ & $2 \cdot 69$ & $1 \cdot 89,3 \cdot 84$ & $<0.0001$ & $1 \cdot 27$ & $0 \cdot 88,1 \cdot 82$ & NS & $2 \cdot 42$ & $1 \cdot 58,3 \cdot 73$ & $<0 \cdot 0001$ \\
\hline \multicolumn{13}{|l|}{ Maternal education } \\
\hline Basic primary & 1.00 & & & 1.00 & & & 1.00 & & & 1.00 & & \\
\hline Higher education & 1.59 & $1 \cdot 16,2 \cdot 20$ & 0.0046 & $1 \cdot 03$ & $0.71,1 \cdot 50$ & NS & 0.75 & $0 \cdot 40,1 \cdot 40$ & NS & $1 \cdot 22$ & $0 \cdot 62,2 \cdot 40$ & NS \\
\hline \multicolumn{13}{|l|}{ Maternal age (years) } \\
\hline$<25 \cdot 0$ & 0.63 & $0 \cdot 45,0 \cdot 89$ & 0.0093 & 0.57 & $0 \cdot 26,1 \cdot 22$ & NS & 0.38 & $0 \cdot 19,0.79$ & 0.0092 & $1 \cdot 14$ & $0 \cdot 47,2 \cdot 74$ & NS \\
\hline $25 \cdot 0-29 \cdot 9$ & 1.00 & & & $1 \cdot 00$ & & & 1.00 & & & 1.00 & & \\
\hline $30 \cdot 0-34 \cdot 9$ & $1 \cdot 60$ & $1 \cdot 26,2 \cdot 01$ & $<0.0001$ & $1 \cdot 35$ & $0.90,2.03$ & NS & 0.83 & $0 \cdot 54,1 \cdot 27$ & NS & $2 \cdot 87$ & $1 \cdot 65,4.99$ & 0.0002 \\
\hline$\geq 35 \cdot 0$ & $1 \cdot 65$ & $1 \cdot 28,2 \cdot 12$ & $<0.0001$ & 1.92 & $1.21,3.06$ & 0.0059 & $1 \cdot 24$ & $0.77,1.99$ & NS & $2 \cdot 93$ & $1 \cdot 63,5 \cdot 27$ & 0.0003 \\
\hline \multicolumn{13}{|l|}{ Pre-pregnancy BMI (kg/m²) } \\
\hline$<18.5$ & $1 \cdot 42$ & $0 \cdot 84,2 \cdot 40$ & NS & 0.75 & $0 \cdot 26,2 \cdot 15$ & NS & 0.56 & $0 \cdot 17,1 \cdot 84$ & NS & $1 \cdot 03$ & $0 \cdot 40,2 \cdot 66$ & NS \\
\hline $18 \cdot 5-24 \cdot 9$ & 1.00 & & & $1 \cdot 00$ & & & $1 \cdot 00$ & & & 1.00 & & \\
\hline$\geq 25 \cdot 0$ & 0.76 & $0.64,0.91$ & 0.0032 & $0 \cdot 81$ & $0.57,1 \cdot 17$ & NS & 0.66 & $0.44,0.98$ & 0.0412 & $1 \cdot 16$ & $0 \cdot 76,1 \cdot 77$ & NS \\
\hline \multicolumn{13}{|l|}{ Smoking during pregnancy } \\
\hline No & 1.00 & & & 1.00 & & & 1.00 & & & 1.00 & & \\
\hline Yes & 0.48 & $0 \cdot 32,0 \cdot 71$ & 0.0002 & 0.95 & $0.54,1.67$ & NS & 0.79 & $0 \cdot 44,1 \cdot 41$ & NS & 0.38 & $0 \cdot 20,0.75$ & 0.0049 \\
\hline \multicolumn{13}{|l|}{ Drinking during pregnancy } \\
\hline No & 1.00 & & & $1 \cdot 00$ & & & 1.00 & & & 1.00 & & \\
\hline Yes & $1 \cdot 17$ & $0 \cdot 97,1 \cdot 40$ & NS & $1 \cdot 01$ & $0 \cdot 71,1 \cdot 45$ & NS & $0 \cdot 82$ & $0 \cdot 55,1 \cdot 23$ & NS & 0.95 & $0 \cdot 63,1 \cdot 44$ & NS \\
\hline \multicolumn{13}{|l|}{ TEDDY child's birth year } \\
\hline $2004 / 2005$ & $1 \cdot 00$ & & & $1 \cdot 00$ & & & $1 \cdot 00$ & & & $1 \cdot 00$ & & \\
\hline 2006 & $1 \cdot 75$ & $1 \cdot 13,2 \cdot 73$ & 0.0128 & $3 \cdot 46$ & $3 \cdot 49,8 \cdot 45$ & 0.0043 & $1 \cdot 80$ & $0 \cdot 88,3 \cdot 66$ & NS & $1 \cdot 81$ & $0.80,4.09$ & NS \\
\hline 2007 & $2 \cdot 69$ & $1 \cdot 80,4 \cdot 07$ & $<0.0001$ & $8 \cdot 70$ & $3 \cdot 87,19 \cdot 55$ & $<0.0001$ & $2 \cdot 87$ & $1 \cdot 51,5 \cdot 48$ & 0.0014 & 2.99 & $1 \cdot 35,6 \cdot 65$ & 0.0070 \\
\hline 2008 & $3 \cdot 71$ & $2 \cdot 47,5 \cdot 56$ & $<0.0001$ & $10 \cdot 07$ & $4 \cdot 48,22 \cdot 66$ & $<0.0001$ & $3 \cdot 46$ & $1 \cdot 80,6 \cdot 63$ & 0.0002 & $2 \cdot 60$ & $1 \cdot 19,5 \cdot 66$ & 0.0165 \\
\hline 2009/2010 & 4.96 & $3 \cdot 33,7 \cdot 40$ & $<0.0001$ & $7 \cdot 70$ & $3 \cdot 43,17 \cdot 30$ & $<0.0001$ & $3 \cdot 42$ & $1 \cdot 82,6 \cdot 45$ & 0.0001 & $4 \cdot 21$ & $1.96,9.04$ & 0.0002 \\
\hline \multicolumn{13}{|l|}{ Race/ethnicity* } \\
\hline Non-Hispanic white & $1 \cdot 00$ & & & & & & & & & & & \\
\hline African Americans & 1.04 & $0.53,2.02$ & NS & & & & & & & & & \\
\hline Hispanic & 0.94 & $0 \cdot 72,1 \cdot 22$ & NS & & & & & & & & & \\
\hline All other & 1.03 & $0.68,1.57$ & NS & & & & & & & & & \\
\hline Missing/unknown & 0.95 & $0.53,1.72$ & NS & & & & & & & & & \\
\hline
\end{tabular}

MVM, multivitamin/mineral.

Separate multivariate models, including the variables listed above, were run for each country.

*US mothers only. 

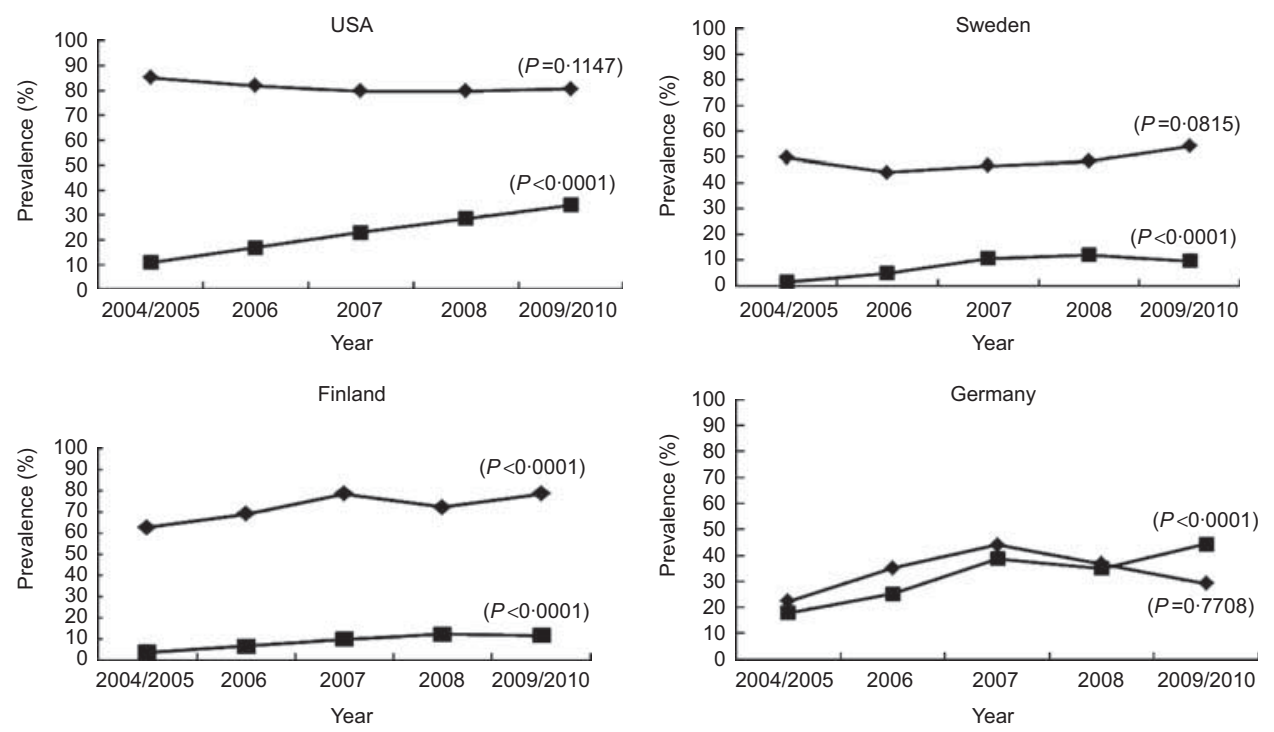

Fig. 1 Trends in reported intake of dietary supplements containing vitamin $D(\longrightarrow)$ and fatty acids $(\longrightarrow-)$ during pregnancy between 2004 and 2010, by country: The Environmental Determinants of Diabetes in the Young (TEDDY) study

that overweight women in Sweden and Germany were less likely to use dietary supplements and overweight women in the USA and Finland were less likely to use supplements containing fatty acids.

One of the few recent studies on maternal supplement use and sociodemographic factors from the USA reports that iron supplementation is associated with ethnicity ${ }^{(23)}$. We did not observe differences regarding race/ethnicity when looking at any supplement use. This may be due to the definition of supplement use, although most of the prenatal multivitamins contain iron. Our description of any dietary supplement use is very broad, it may contain supplement use for health-seeking reasons (enhance the diet) and for medical reasons (e.g. anaemia). We do not have information about the reason why women use dietary supplements and are therefore not able to separate these behaviours. However, when looking at vitamin Dcontaining supplements, women with race/ethnicity other than non-Hispanic white were less likely to use these supplements.

The variable maternal education only affected women in the USA and Sweden. It has been implied that a higher education level gives a greater awareness of the role of nutrition in good health ${ }^{(24,25)}$. Factors such as being pregnant with the first child and higher maternal age may also indicate higher awareness regarding possible mechanisms in diet-related health. In our study, the use of supplements containing fatty acids may give a better picture of a health-seeking behaviour during pregnancy. An adequate intake of DHA in pregnant women is critical to development of the fetal brain in the third trimester ${ }^{(26)}$, and has also been linked to reduced risk of T1D in the offspring ${ }^{(4)}$. The recommended DHA intake should preferably come from consumption of one or two fish meals per week or a dietary supplement ${ }^{(27)}$. However, despite higher needs during pregnancy, women often decrease fish consumption due to warnings about contaminants such as methyl mercury and dioxins. Reduced or altered fish consumption together with low frequency of fatty acid supplementation may lead to insufficient exposure to long-chain $n$-3 fatty acids in utero ${ }^{(27)}$. The use of fatty acid supplementation during pregnancy was not common in the TEDDY population, with less than $10 \%$ in Finland and Sweden but higher in the USA (24\%) and Germany (32\%). In Norway, a Nordic country with a long-standing tradition of cod-liver oil use, the prevalence of fatty acid supplement use was $59 \%$ among pregnant women ${ }^{(1)}$. The lower prevalence in TEDDY could be compared with another Nordic country, Denmark, where only $4 \%$ of women reported use of fish oil formulations during pregnancy $^{(28)}$. The use of fatty acid supplementation has increased in all TEDDY countries. Birth year of the child was a factor that was significant across all countries. The later the child was born during the TEDDY screening period, the more likely it was that the mother used fatty acid supplements. This could reflect different trends in the prenatal supplement markets, but could also be due to revised national recommendations.

There are very few studies on prevalence of supplement use during pregnancy in the USA, but in a study on pregnant women in California during 2006-2008, 89\% of the women reported using dietary supplements ${ }^{(2)}$. More studies from Europe have presented data on maternal supplement use. In the DIPP study in 1998-2000, $85 \%$ of the women reported taking dietary supplements ${ }^{(9)}$. In the All Babies in Southeast Sweden (ABIS) study in Sweden, $56 \%$ of the women took some type of dietary supplement during pregnancy in 1997-1999 ${ }^{(13)}$; this can be compared with, almost 10 years later, the $89 \%$ of Swedish women in TEDDY who reported any supplement use. The high 
Table 6 Country-specific dietary supplement recommendations for pregnant women (TEDDY screening period 2004-2010)

\begin{tabular}{|c|c|c|c|c|}
\hline & $U_{S A}^{*(8)}$ & Sweden ${ }^{(30)}$ & Finland $^{(31)}$ & Germany $^{(29)}$ \\
\hline Folic acid & $\begin{array}{l}400 \mu \mathrm{g} / \mathrm{d} \text { during first } \\
\text { trimester }\end{array}$ & $400 \mu \mathrm{g} / \mathrm{d}$ during first trimester & $\begin{array}{l}400 \mu \mathrm{g} / \mathrm{d} \text { during first } \\
\text { trimester }\end{array}$ & $400 \mu \mathrm{g} / \mathrm{d}$ during first trimester \\
\hline Iron & $27 \mathrm{mg} / \mathrm{d}$ & $\begin{array}{l}100 \mathrm{mg} / \mathrm{d} \text { in second half of } \\
\text { pregnancy - recommendation } \\
\text { removed in } 2008\end{array}$ & & \\
\hline lodine & & & & $100-(150) \mu \mathrm{g} / \mathrm{d}$ \\
\hline Vitamin D & & & $\begin{array}{l}10 \mu \mathrm{g} / \mathrm{d} \text { during } \\
\text { winter months }\end{array}$ & \\
\hline DHA $(22: 6 n-3)$ & & $\begin{array}{l}\text { Women who do not eat fish: } \\
200 \mathrm{mg} / \mathrm{d}-\text { recommendation } \\
\text { since } 2008\end{array}$ & & $\begin{array}{l}\text { Women who do not eat fish: } \\
200 \mathrm{mg} / \mathrm{d}-\text { recommendation } \\
\text { since } 2008\end{array}$ \\
\hline
\end{tabular}

TEDDY, The Environmental Determinants of Diabetes in the Young.

*A prenatal multivitamin/multimineral supplement is recommended for women with iron-deficiency anaemia, poor-quality diets, vegans, vegetarians, smokers and women carrying two or more fetuses.

prevalence of supplement use in all TEDDY countries may be attributed to the recommendations on supplementation during pregnancy. The folic acid recommendation of $400 \mu \mathrm{g} / \mathrm{d}$ before getting pregnant and for the first trimester is given in all countries. Most countries recommend iron supplementation if the status is too low, often during the second half of the pregnancy. In the USA, health-care providers recommend women to use a standard prenatal multivitamin/multimineral supplement throughout pregnancy and even before becoming pregnant ${ }^{(8)}$. In Europe, there are variations in additional supplement recommendations and they have changed during the TEDDY study period. In Germany, prenatal iodine supplementation is recommended ${ }^{(29)}$. Since 2008, both Germany and Sweden have clarified the benefits of eating fish during pregnancy ${ }^{(29,30)}$ in their national recommendations. Women who do not eat fish are recommended to eat $200 \mathrm{mg}$ DHA/d during pregnancy. In Finland, pregnant women are currently recommended to take $10 \mu \mathrm{g}$ of supplemental vitamin D daily throughout the year; before 2011, vitamin D supplements were recommended to be used only during winter months ${ }^{(31)}$. Table 6 summarizes the country-specific recommendations regarding dietary supplementation during pregnancy. Country-specific recommendations on vitamin $\mathrm{D}$ and fatty acids were reflected in our data. Countries with vitamin D recommendations had higher prevalence of supplement use than countries without specific recommendations.

Our findings may not be readily generalized to the general population due to our selection criterion of high-risk HLA genotypes representing approximately $5 \%$ of the population, but the results are important and comparable with other longitudinal studies.

\section{Conclusion}

The present study captured a high prevalence of supplement use during pregnancy in the USA, Sweden, Finland and Germany. Being pregnant with the first child was a strong predictor for any supplement use in all countries.
Higher education, low maternal age, $B M I \geq 25 \cdot 0 \mathrm{~kg} / \mathrm{m}^{2}$ and smoking during pregnancy were additional predictors but not consistently across all countries. The use of vitamin D-containing supplements was more common in countries with pertinent national recommendations.

\section{Acknowledgements}

Sources of funding: The TEDDY study was funded by grant numbers DK 63829, 63861, 63821, 63865, 63863, 63836, 63790 and UC4DK095300 and contract number HHSN267200700014C from the National Institute of Diabetes and Digestive and Kidney Diseases (NIDDK), the National Institute of Allergy and Infectious Diseases (NIAID), the National Institute of Child Health and Human Development (NICHD), the National Institute of Environmental Health Sciences (NIEHS), the Juvenile Diabetes Research Foundation (JDRF) and the Centers for Disease Control and Prevention (CDC). Conflicts of interest: The authors declare no conflict of interest. Authors' contributions: C.A.A. wrote the manuscript and analysed the data; K.V. did the statistical analysis; J.Y. and U.U. assisted with drafting the manuscript and critically reviewed the manuscript; K.H., G.J. and A.R. critically reviewed the manuscript; L.B. was responsible for data handling; and S.V. and J.N. designed the study and critically reviewed the manuscript; J.M.N. and S.M.V. share last authorship. Acknowledgements: The participation of all families in the TEDDY study is gratefully acknowledged.

\section{References}

1. Haugen M, Brantsæeter A, Alexander J et al. (2008) Dietary supplements contribute substantially to the total nutrient intake in pregnant Norwegian women. Ann Nutr Metab 52, 272-280.

2. Dror D, King J, Durand D et al. (2011) Association of modifiable and nonmodifiable factors with vitamin D status in pregnant women and neonates in Oakland CA. $J$ Am Diet Assoc 111, 111-116.

3. Brantsæeter A, Haugen M, Hagve TA et al. (2007) Selfreported dietary supplement use is confirmed by biological markers in the Norwegian Mother and Child Cohort study (MoBa). Ann Nutr Metab 51, 146-154. 
4. Stene LC, Ulriksen J, Magnus P et al. (2000) Use of cod liver oil during pregnancy associated with lower risk of type 1 diabetes in the offspring. Diabetologia 43, 1093-1098.

5. Fronczak CM, Baron A, Chase PH et al. (2003) In utero dietary exposures and risk of islet autoimmunity in children. Diabetes Care 26, 3237-3242.

6. Hewison M (2012) An update on vitamin D and human immunity. Clin Endocrinol (Oxf) 76, 315-325.

7. Calder PC (2007) Immunomodulation by omega-3 fatty acids. Prostaglandins Leukot Essent Fatty Acids 77, 327-335.

8. Kaiser L, Allen L \& ADA Reports (2008) Position of the American Dietetic Association: nutrition and lifestyle for healthy pregnancy outcome. J Am Diet Assoc 108, 553-561.

9. Arkkola T, Uusitalo U, Pietikäinen M et al. (2006) Dietary intake and use of dietary supplements in relation to demographic variables among pregnant Finnish women. Br J Nutr 96, 913-920.

10. Picciano M \& McGuire M (2009) Use of dietary supplements by pregnant women and lactating women in North America. Am J Clin Nutr 89, issue 2, 663S-667S.

11. Bailey R, Gahche J, Lentino C et al. (2011) Dietary supplement use in the United States, 2003-2006. J Nutr 141, 261-266.

12. Rock CL (2007) Multivitamin-multimineral supplements: who uses them? Am J Clin Nutr 85, issue 1, 277S-279S.

13. Brekke HK \& Ludvigsson J (2007) Vitamin D supplementation and diabetes related autoimmunity in the ABIS study. Pediatr Diabetes 8, 11-14.

14. Marjamäki L, Niinistö S, Kenward MG et al. (2010) Maternal intake of vitamin D during pregnancy and risk of advanced $\beta$ cell autoimmunity and type 1 diabetes in offspring. Diabetologia 8, 1599-1607.

15. TEDDY Study Group (2008) The Environmental Determinants of Diabetes in the Young (TEDDY) study. Ann NY Acad Sci 1150, 1-13.

16. Hagopian WA, Erlich H, Lernmark $\AA$ et al. (2011) The Environmental Determinants of Diabetes in the Young (TEDDY): genetic criteria and international diabetes risk screening of 421000 infants. Pediatr Diabetes 12, 733-743.

17. The TEDDY Study Group (2007) The Environmental Determinants of Diabetes in the Young (TEDDY) study: study design. Pediatr Diabetes 8, 286-298.

18. US Food and Drug Administration (2009) Overview of Dietary Supplements. http://fda.gov/Food/DietarySupplements/ ConsumerInformation/ucm110417.htm (accessed October 2009).

19. Moyers S, Richesson R \& Krischer J (2008) Trans-Atlantic data harmonization in the classifications of medicines and dietary supplements: a challenge for epidemiologic study and clinical research. Int J Med Inform 77, 58-67.

20. World Health Organization (2000) Obesity: Preventing and Managing the Global Epidemic. Report of a WHO Consultation. WHO Technical Report Series no. 894. Geneva: WHO.

21. Burton A, Wilson S \& Gillies AJ (2001) Folic acid: is self-reported use of supplements accurate? J Epidemiol Community Health 55, 841-842.

22. Brustad M, Braaten T \& Lund E (2004) Predictors for cod-liver oil supplement use - the Norwegian Women and Cancer Study. Eur J Clin Nutr 58, 128-136.

23. Cogswell ME, Kettel-Khan L \& Ramakrishnan U (2003) Iron supplement use among women in the United States: science, policy and practice. J Nutr 133, issue 6, 1974S-1977S.

24. Foote JA, Murphy SP, Wilkens L et al. (2003) Factors associated with dietary supplement use among healthy adults of five ethnicities. The Multiethnic Cohort Study. Am J Epidemiol 157, 888-897.

25. Conner M, Kirk S, Cade J et al. (2003) Environmental influences: factors influencing a woman's decision to use dietary supplements. J Nutr 133, issue 6, 1978S-1982S.
26. Zeisel S (2009) Is maternal diet supplementation beneficial? Optimal development of infants depends on mother's diet. Am J Clin Nutr 89, issue 2, 685S-687S.

27. Koletzko B, Cetin I \& Brenna JT; for the Perinatal Lipid Intake Working Group (2007) Dietary fat intakes for pregnant and lactating women. Br J Nutr 98, 873-877.

28. Olsen SF, Mikkelsen TB, Knudsen VK et al. (2007) Data collected on maternal dietary exposures in the Danish National Birth Cohort. Paediatr Perinat Epidemiol 21, 76-86.

29. German Nutrition Society, Austrian Nutrition Society, Swiss Society for Nutrition Research \& Swiss Nutrition Association (2012) D-A-CH Referenzwerte für die Nährstoffzufuhr, 1st ed, 4th corrected reprint. Rostock: DGE-MedienService.

30. Livsmedelsverket (2008) Näringsämnen vid graviditet och amning. Rapport 26 - 2008. Uppsala: Livsmedelsverket.

31. Finnish Nutrition Council (2011) Nutrient recommendations. http://www.ravitsemusneuvottelukunta.fi/portal/fi/ tiedotteet_ja_kannanotot/ (accessed January 2011).

\section{Appendix 1}

\section{The TEDDY Study Group}

\section{Colorado Clinical Center}

Marian Rewers, MD, PhD, Principal Investigator ${ }^{1,4,6,10,11}$; Katherine Barriga $^{12}$; Kimberly Bautista ${ }^{12}$; Judith Baxter ${ }^{9,12,15}$; George Eisenbarth, MD, PhD; Nicole Frank ${ }^{2}$; Patricia Gesualdo $^{2,6,12,14,15}$; Michelle Hoffman ${ }^{12,13,14}$; Lisa Ide; Rachel Karban $^{12}$; Edwin Liu, MD ${ }^{13}$; Jill Norris, $\mathrm{PhD}^{2,12}$; Kathleen Waugh $^{7,12,15}$; Adela Samper-Imaz; Andrea Steck, MD (University of Colorado, Anschutz Medical Campus, Barbara Davis Center for Childhood Diabetes).

\section{Georgia/Florida Clinical Center}

Jin-Xiong She, PhD, Principal Investigator ${ }^{\mathrm{a}, 1,3,4,11}$; Desmond Schatz, MD ${ }^{\mathrm{b}, 4,5,7,8} ;$ Diane Hopkins ${ }^{\mathrm{a}, 12}$; Leigh Steed $^{\mathrm{a}, 12,13,14,15}$; Jamie Thomas ${ }^{\mathrm{b}, 6,12}$; Katherine Silvis ${ }^{\mathrm{a}, 2}$; Michael Haller, MD ${ }^{\mathrm{b}, 14}$; Meena Shankar ${ }^{\mathrm{b}, 2}$; Kim English ${ }^{\mathrm{a}}$; Richard McIndoe, $\mathrm{PhD}^{\mathrm{a}}$; Haitao Liu, MD ${ }^{\mathrm{c}}$; John Nechtman ${ }^{\mathrm{c}}$; Ashok Sharma ${ }^{\mathrm{a}}$; Joshua Williams ${ }^{\mathrm{a}}$; Gabriela Foghis ${ }^{\mathrm{a}}$; Stephen W. Anderson, $\mathrm{MD}^{\mathrm{d}}$ ( ${ }^{\mathrm{a}}$ Georgia Health Sciences University; bUniversity of Florida; 'Jinfiniti Biosciences LLC, Augusta, GA; ${ }^{\mathrm{d}}$ Pediatric Endocrine Associates, Atlanta, GA).

\section{Germany Clinical Center}

Anette G. Ziegler MD, Principal Investigator ${ }^{\mathrm{a}, 1,3,4,11}$; Alexandra Achenbach, $\mathrm{PhD}^{\mathrm{b}, 12}$; Andreas Beyerlein, $\mathrm{PhD}^{\mathrm{a}, 2}$; Ezio Bonifacio, $\mathrm{PhD}^{\mathrm{b}, 5}$; Nadine Bruckmeier ${ }^{\mathrm{a}}$; Melanie Bunk ${ }^{\mathrm{a}}$; Vanessa Dietrich ${ }^{\mathrm{a}}$; Anna Huppert, MD ${ }^{\mathrm{a}}$; Minna Harsunen, MD ${ }^{\mathrm{a}}$; Lydia Henneberger ${ }^{\mathrm{a}, 2,12}$; Michael Hummel, $\mathrm{MD}^{\mathrm{a}, 13}$; Sandra Hummel, $\mathrm{PhD}^{\mathrm{a}, 2}$; Gesa Joslowski $^{\mathrm{c}, 2}$; Mathilde Kersting, $\mathrm{PhD}^{\mathrm{c}, 2}$; Annette Knopff ${ }^{\mathrm{a}, 7}$; Nadja Kocher ${ }^{\mathrm{a}}$; Sibylle Koletzko, MD ${ }^{\mathrm{d}, 13}$; Miriam Krasmann, MD ${ }^{\mathrm{a}}$; Stephanie Krause ${ }^{\mathrm{a}}$; Lorenz Lachmann ${ }^{\mathrm{a}}$; Claudia Matzke; ; Claudia Peplow $^{\mathrm{b}, 12}$; Maren Pflüger, $\mathrm{PhD}^{\mathrm{a}, 6}$; Claudia Ramminger ${ }^{\mathrm{a}}$; Christiane Ried ${ }^{\mathrm{a}}$; Roswith Roth, PhD ${ }^{\mathrm{a}, 9}$; Julia Schenkel ${ }^{\mathrm{a}}$; Marlon Scholz ${ }^{\mathrm{a}}$; Joanna Stock $^{\mathrm{a}}$; Elisabeth Strauss ${ }^{\mathrm{a}}$; Alexandra Thalmeier ${ }^{\mathrm{a}}$; Katja Voit ${ }^{\mathrm{a}}$; Katharina Warncke, $\mathrm{MD}^{\mathrm{a}, 14}$; Christiane Winkler, $\mathrm{PhD}^{\mathrm{a}, 2,12,15}$; 
Anja Wosch ${ }^{\mathrm{a}}$ ( ${ }^{\mathrm{a}}$ Forschergruppe Diabetes eV at Helmholtz Zentrum München; ${ }^{b}$ Center for Regenerative Therapies, TU Dresden; 'Research Institute for Child Nutrition, Dortmund; ${ }^{\mathrm{d} D r}$. von Hauner Children's Hospital, Department of Gastroenterology, Ludwig Maximillians University Munich).

\section{Finland Clinical Center}

Olli G. Simell, MD, PhD, Principal Investigator ${ }^{\mathrm{a}, \mathrm{b}, 1,4,11,13}$; Heikki Hyöty, MD, $\mathrm{PhD}^{\mathrm{c}, \mathrm{d}, 6} ;$ Jorma Ilonen, $\mathrm{MD}, \mathrm{PhD}^{\mathrm{a}, \mathrm{e}, 3}$; Mikael Knip, MD, $\mathrm{PhD}^{\mathrm{c}, \mathrm{d}}$; Maria Lonnrot, $\mathrm{MD}, \mathrm{PhD}^{\mathrm{c}, \mathrm{d}, 6}$; Elina Mantymaki ${ }^{\mathrm{a}, \mathrm{b}}$; Juha Mykkänen, $\mathrm{PhD}^{\mathrm{a}, \mathrm{b}, 3}$; Kirsti Nanto-Salonen, MD, $\mathrm{PhD}^{\mathrm{a}, \mathrm{b}, 12}$; Tiina Niininen ${ }^{\mathrm{c}, \mathrm{d}}$; Mia Nyblom $^{\mathrm{c}, \mathrm{d}}$; Anne Riikonen ${ }^{\mathrm{c}, \mathrm{d}, 2}$; Minna Romo ${ }^{\mathrm{a}, \mathrm{b}}$; Barbara Simell ${ }^{\mathrm{a}, \mathrm{b}, 12,15}$; Tuula Simell, PhD ${ }^{\mathrm{a}, \mathrm{b}, 9,12}$; Ville Simell ${ }^{\mathrm{a}, \mathrm{b}, 13}$; Maija Sjöberg ${ }^{\mathrm{a}, \mathrm{b}, 12,14}$; Aino Stenius ${ }^{\mathrm{f}, \mathrm{g}}$; Eeva Varjonen ${ }^{\mathrm{a}, \mathrm{b}}$; Riitta Veijola, MD, $\mathrm{PhD}^{\mathrm{f}, \mathrm{g}}$; Suvi M. Virtanen, $\mathrm{MD}, \mathrm{PhD}^{\mathrm{c}, \mathrm{d}, \mathrm{h}, 2}$ ( ${ }^{\mathrm{a}}$ University of Turku; ${ }^{\mathrm{b}}$ Turku University Hospital; ${ }^{\mathrm{C}}$ University of Tampere; ${ }^{\mathrm{d}}$ Tampere University Hospital; ${ }^{\mathrm{e}}$ University of Kuopio; ${ }^{\mathrm{f}}$ University of Oulu; ${ }^{\mathrm{g}}$ Oulu University Hospital; ${ }^{\mathrm{h}}$ National Institute for Health and Welfare, Finland).

\section{Sweden Clinical Center}

Åke Lernmark, PhD, Principal Investigator ${ }^{1,3,4,8,10,11,15}$; Daniel Agardh, MD, $\mathrm{PhD}^{13}$; Peter Almgren; Eva Andersson; Carin Andrén-Aronsson ${ }^{2,13}$; Maria Ask; Ulla-Marie Karlsson; Corrado Cilio, MD, $\mathrm{PhD}^{5}$; Jenny Bremer; Emilie Ericson-Hallström; Thomas Gard; Joanna Gerardsson; Gertie Hansson ${ }^{12,14}$; Monica Hansen; Susanne Hyberg; Rasmus Håkansson; Fredrik Johansen; Linda Jonsson; Helena Larsson, MD, $\mathrm{PhD}^{14}$; Barbro Lernmark, $\mathrm{PhD}^{9,12}$; Maria Markan; Theodosia Massadakis; Jessica Melin; Maria Månsson-Martinez; Anita Nilsson; Kobra Rahmati; Monica Sedig Järvirova; Sara Sibthorpe; Birgitta Sjöberg; Carina Törn, $\mathrm{PhD}^{3,15}$; Anne Wallin; Åsa Wimar; Sofie Åberg (Lund University).

\section{Washington Clinical Center}

William A. Hagopian, MD, PhD, Principal Investigator $^{1,3,4,5,6,7,11,13,14}$; Xiang Yan, MD; Michael Killian $^{6,7,12,13}$; Claire Cowen Crouch ${ }^{12,14,15}$; Kristen M. Hay ${ }^{2}$; Stephen Ayres; Carissa Adams; Brandi Bratrude; David Coughlin; Greer Fowler; Czarina Franco; Carla Hammar; Diana Heaney; Patrick Marcus; Arlene Meyer; Denise Mulenga; Elizabeth Scott; Jennifer Skidmore; Joshua Stabbert; Viktoria Stepitova; Nancy Williams (Pacific Northwest Diabetes Research Institute).

\section{Pennsylvania Satellite Center}

Dorothy Becker, MD; Margaret Franciscus ${ }^{12}$; MaryEllen Dalmagro-Elias ${ }^{2}$; Ashi Daftary, MD (Children's Hospital of Pittsburgh, University of Pittsburgh Medical Center).

\section{Data Coordinating Center}

Jeffrey P. Krischer, PhD, Principal Investigator ${ }^{1,4,5,10,11}$; Michael Abbondondolo; Lori Ballard ${ }^{3,9,14,15}$; Rasheedah
Brown $^{12,15}$; Brant Burkhardt, PhD ${ }^{5,6}$; David Cuthbertson; Christopher Eberhard; Steven Fiske; Veena Gowda; David Hadley, $\mathrm{PhD}^{3,13}$; Hye-Seung Lee, $\mathrm{PhD}^{3,6,13,15}$; Shu Liu; Kristian Lynch, $\mathrm{PhD}^{9}$; Jamie Malloy; Cristina McCarthy $^{12,15}$; Wendy McLeod ${ }^{2,5,6,13,15}$; Laura Smith, PhD ${ }^{9}$; Susan Smith ${ }^{12,15}$; Ulla Uusitalo, $\mathrm{PhD}^{2,15}$; Kendra Vehik, $\mathrm{PhD}^{4,5,9,14,15}$; Earnest Washington; Jimin Yang, PhD, RD ${ }^{2,15}$ (University of South Florida).

\section{Project Scientist}

Beena Akolkar, PhD ${ }^{1,3,4,5,7,10,11}$ (National Institutes of Diabetes and Digestive and Kidney Diseases).

\section{Other contributors}

Kasia Bourcier, $\mathrm{PhD}^{\mathrm{a}, 5}$; Thomas Briese, $\mathrm{PhD}^{\mathrm{b}, 6,15}$; Henry Erlich, $\mathrm{PhD}^{\mathrm{c}, 3}$; Suzanne Bennett Johnson, $\mathrm{PhD}^{\mathrm{d}, 9,12}$; Steve Oberste, $\mathrm{PhD}^{\mathrm{e}, 6}$ ( ${ }^{\mathrm{a}}$ National Institutes of Allergy and Infectious Diseases; ${ }^{\mathrm{b} C o l u m b i a}$ University; ${ }^{\mathrm{c} C h i l d r e n ' s ~ H o s p i t a l ~ O a k l a n d ~}$ Research Institute; ${ }^{\mathrm{d}}$ Florida State University; ${ }^{\mathrm{e}}$ Centers for Disease Control and Prevention).

\section{Committees}

${ }^{1}$ Ancillary Studies; ${ }^{2}$ Diet; ${ }^{3}$ Genetics; ${ }^{4}$ Human Subjects/ Publicity/Publications; ${ }^{5}$ Immune Markers; ${ }^{6}$ Infectious Agents; ${ }^{7}$ Laboratory Implementation; ${ }^{8}$ Maternal Studies; ${ }^{9}$ Psychosocial; ${ }^{10}$ Quality Assurance; ${ }^{11}$ Steering; ${ }^{12}$ Study Coordinators; ${ }^{13}$ Celiac Disease; ${ }^{14}$ Clinical Implementation; ${ }^{15}$ Quality Assurance Subcommittee on Data Quality.

\section{Appendix 2}

\section{Coding of dietary supplements in The Environmental Determinants of Diabetes in the Young (TEDDY) study}

Single vitamins and minerals

- Vitamin D

- Vitamin C

- Probiotic

- Single fatty acid (e.g. DHA, EPA, $\gamma$-linolenic acid, dihomo- $\gamma$-linolenic acid, arachidonic acid)

- Calcium

- Vitamin $\mathrm{B}_{6}$

- Vitamin $\mathrm{B}_{12}$

- Folic acid

- Vitamin A

- Vitamin E

- Iron

- Niacin

- Zinc

- Magnesium

- Potassium

- Choline

- Vitamin $B_{1}$

- Vitamin $\mathrm{B}_{2}$ 
- Biotin

- Pantothenic acid

- Selenium

- Chromium

- Fluorine

- $\beta$-Carotene

- Iodine

- Copper

- Antioxidants (non-vitamin/mineral; e.g. lycopene, coenzyme $\left.\mathrm{Q}_{10}\right)$

\section{Multivitamins/minerals}

- Multivitamins/minerals with probiotic (no vitamin D, no fish oil/fatty acids)

- Multivitamins/minerals containing vitamin D (no probiotic, no fish oil/fatty acids)
- Multivitamins/minerals (no vitamin D, no probiotic, no fish oil/fatty acids)

- Multivitamins/minerals with fish oil/fatty acids (no vitamin D, no probiotic)

- Multivitamins/minerals with fish oil/fatty acids containing vitamin D (no probiotic)

- Multivitamins/minerals with probiotic and fish oil/fatty acids and containing vitamin D

- Multivitamins/minerals with probiotic containing vitamin D (no fish oil/fatty acids)

- Multivitamins/minerals with probiotic and fish oil/fatty acids (no vitamin D)

- Antioxidants (products with several non-vitamin/ mineral antioxidants, e.g. combination of lycopene, coenzyme $\mathrm{Q}_{10}$, etc.)

- Mixture of fatty acids without vitamin/mineral (no probiotic, no vitamin D) 